\title{
Stochastic Traffic Engineering for Live Audio/Video Delivering over Energy-Limited Wireless Access Networks
}

\author{
Nicola Cordeschi`, Tatiana Patriarca, and Enzo Baccarelli \\ DIET Dept.,"Sapienza" University of Rome \\ via Eudossiana 18, 00184 Rome, Italy \\ Tel.: +39 06 44585366; Fax: +39 064873300 \\ \{cordeschi, patriarca, enzobac\}@infocom . uniroma1.it
}

\begin{abstract}
We study the Stochastic Traffic Engineering (STE) problem arising from the support of QoS-demanding live (e.g., real time) audio/video applications over unreliable IP-over-wireless access pipes. First, we recast the problem to be tackled in the form of a suitable nonlinear stochastic optimization problem, and then we develop a goodput analysis for the resulting IP-over-wireless pipe that points out the relative effects of fading-induced errors and congestion-induced packet's losses. Second, we present an optimal resource-management policy that allows a joint scheduling of playin, transmit and playout rates. Salient features of the developed joint scheduling policy are that: i) it is self-adaptive; and, ii) it is able to implement reliable Constant Bit Rate (CBR) connections on the top of unreliable energy-limited wireless pipes.
\end{abstract}

Keywords: IP-over-wireless connections, real-time streaming, Stochastic Traffic Engineering (STE), self-adaptive rate-control, hard QoS guarantees.

\section{Introduction and Related Works}

Due to the unreliable randomly time-varying transport quality currently offered by IP-over-wireless connections, providing QoS guarantees to real-time media applications (e.g., live audio/video) over energy-limited congestion-prone wireless IP domains is a (still open) challenging task, that requires an optimized (possibly, self-adaptive) management of the floating resources done available by the underlying pipe [1. In principle, a suitable means to approach this target is provided by the optimization framework and self-adaptive controlling mechanisms offered by Traffic Engineering (TE). Differently from the (more) usual QoS resource's allocation, the TE solution not only aims to guarantee specified QoS levels, but also optimizes an overall system-wide performance metric, mainly by performing joint flow-control at the source host, destination host and switching (e.g., serving) nodes. This more conventional TE approach evolves to

\footnotetext{
^ Corresponding author.
} 


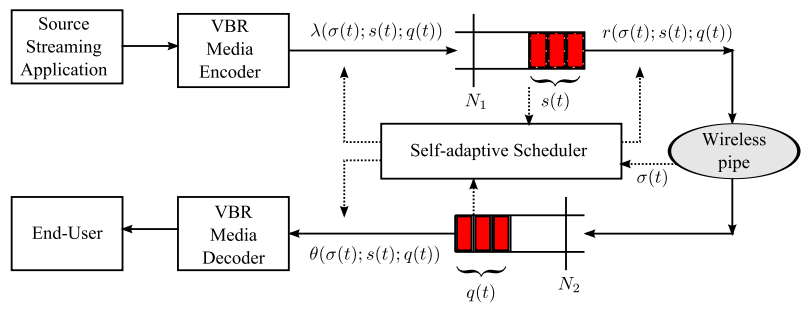

Fig. 1. The considered battery-powered wireless streaming system

the Stochastic TE (STE) one [2, ,3, when the underlying connection is affected by some form of uncertainty, as, for example, the random behavior of the state of the IP-over-wireless pipe in the (here considered) streaming scenario of Fig 1 . Hence, triggered by these considerations, in this work we resort to the STE principles for designing a joint self-adaptive playin-energy-playout scheduler for the streaming system of Fig[1. Specifically, as sketched in Fig 1, we model the (possibly mobile) battery-powered (i.e., energy-limited) source node (e.g., the Source Host (SH)) as a time-slotted fluid G/G/1 queue fed by a Variable Bit Rate (VBR) media encoder, whose output rate (e.g., the playin rate) may be adaptively controlled. Analogously, the receive node (e.g., the Destination Host $(\mathrm{DH})$ ) is equipped with a G/G/1 queue, whose output rate (e.g., the playout rate) may be also controlled. In principle, both queues may be considered implemented at the APPlication (APP) layers of the underlying protocol stack, so that the corresponding wireless connection of Fig 1 models the overall resulting peer-to-peer virtual pipe.

The considered system-wide perform metric to be maximized is the resulting transmit rate averaged over both the fading and congestion-induced statistics of the state of the wireless pipe, under seven system's constraints. The first one is on the available average energy per slot and it arises from energy limitations typically imposed by the PHY layer of the considered modems. The second and third constraints upper limit the available buffer capacities, while the fourth constraint arises from the APP layer and fixes the maximum instantaneous playin rate. Fifth constraint upper bounds the pre-roll delay [1], while sixth and seventh constraints introduce hard (e.g., deterministic) upper and lower bounds on the instantaneous values allowed the playout rate.

Optimized schedulers derived by exploiting the analytical tools of Nonlinear Optimization and Queueing Theory and, thus, implemented via TE-based controlling mechanisms are presented, for example, in [4, [5, 6]. In [4, authors develop an optimized policy for controlling both transmit power and playout rate of the considered streaming system, the target being that to minimize the average power consumption and maximize the rendered average media quality. More recently, some STE aspects related to the wireless access are tackled in [5], [6]. However, they do not consider the control of the playout rate, neither account for (possible) limitations on the available average energy-budget. In the application context of multimedia systems and services, Adaptive Media Playout (AMP) policies aiming at minimizing suitable rate-distortion functions are the 
focus of the contributions in [8, 13. However, contributions in [8, [13] are not STE-oriented, so that they do not consider the adaptive control of the playin rate and/or transmit energy, and (which is the most) do not provide hard QoS guarantees to the supported media applications.

\section{QoS Real-Time Streaming: System and Traffic Modeling}

In the streaming system of Fig[1] time is slotted, with slot-duration of $T_{s}$ (sec.) and the $t$-th slot spans the (semi-open) interval $[t,(t+1)), t \in \mathbb{N}_{0}^{+}$. The APP layers at the source and destination hosts of Fig 1 are equipped with playin and playout buffers of finite capacities $N_{1}$ and $N_{2}$, respectively. The number of information units (IUs) arriving at the input of the playin buffer at the end of slot $t$ is the playin rate $\lambda(t) \in \mathbb{R}_{0}^{+}(I U /$ slot $)$ at slot $t$. The underlying wireless physical channel supporting the peer-to-peer pipe of Fig 1 is affected by interference, noise and fading phenomena, we assume constant over each slot (i.e., we assume an ergodic "block fading" physical channel [12]). Hence, the resulting state $\boldsymbol{\sigma}(t) \in$ $\mathbb{R}_{0}^{+}$at the $t$-th slot of the overall pipe is modelled as a real-valued nonnegative r.v. with (a priori unknown) steady-state pdf $p_{\sigma}(\sigma) 11$ Furthermore, the connection state value $\sigma(t)$ is assumed to be known at the $\mathrm{SH}$ at the beginning of slot $t$. The overall streaming system is considered to operate under stationary and ergodic conditions (i.e., it works in the steady-state). About backlog dynamics of the playin/playout queues, let $s(t) \in \mathbb{R}_{0}^{+}$be the number of IUs buffered by playin queue of Fig 1 at the beginning of slot $t$ (i.e., the backlog of the playin buffer). Thus, after denoting by $r(t)(I U /$ slot $)$ the number of IUs to be sent over the wireless connection during $t$-th slot, the following Lindley's equation:

$$
s(t+1)=[s(t)+\lambda(t)-r(t)]^{+}, t \geq 0,
$$

dictates the evolution of the discrete-time backlog process $\left\{s(t) \in \mathbb{R}_{0}^{+}, t \geq 0\right\}$ at the playin queue. Let $q(t) \in \mathbb{R}_{0}^{+}$be the number of IUs present in the playout buffer at the beginning of slot $t$ (i.e., the backlog of the playout buffer). Hence, after denoting by $\theta(t)(I U /$ slot $)$ the number of IUs rendered to the VBR media decoder of Fig 1 at the end of slot $t$, the evolution of the playout backlog is described by the corresponding Lindley's equation:

$$
q(t+1)=[q(t)+r(t)-\theta(t)]^{+}, t \geq 0 .
$$

The cost of sending $r(t)$ IUs over slot $t$ is the amount of energy $\mathcal{E}(t)$ (measured in Joule $(J))$ required for their transmission. Thus, we assume that $r(t)$ depends on both $\mathcal{E}(t)$ and $\sigma(t)$ via the rate-function $\mathcal{R}(\cdot ; \cdot)$ adopted to measure the (instantaneous) goodput of the considered connection, so that we can write

$$
r(t) \triangleq \mathcal{R}(\mathcal{E}(t) ; \sigma(t)), \quad t \geq 1 .
$$

${ }^{1}$ The meaning of the connection state $\sigma(t)$ is application depending. An example of $\sigma(t)$ modelling the overall behavior of a TCP-friendly connection over an IP-based wireless access network is detailed in Sect 4 . 
Since $\mathcal{R}(\cdot ; \cdot)$ summarizes the goodput-performance of the end-to-end virtual pipe of Fig, 1, its actual behavior and structural properties are system depending. Therefore, we limit to introduce few (quite mild) assumptions on $\mathcal{R}(\cdot ; \cdot)$, that, by fact, are retained by rate-functions of practical interest [1]. We just assume that it is nondecreasing for $\mathcal{E} \geq 0$ and $\sigma \geq 0$ and, for any assigned $\sigma \neq 0$, the rate-function is strictly concave in the $\mathcal{E}$-variable.

\subsection{QoS Support for Real-Time Streaming}

The analytical expression assumed by the distortion-function $D(\theta): \mathbb{R}_{0}^{+} \rightarrow \mathbb{R}_{0}^{+}$ adopted to measure the (subjective) level of satisfaction perceived by the enduser of Fig 1 is strongly application depending, and no general formulae are available for it [1, 13. Therefore, according to an emerging trend [1, we (only) assume that the adopted distortion-function $D(\theta)$ is a real, nonnegative, strictly decreasing function of the actually experimented playout rate $\theta$ [1], 13]. This means that, after denoting by $D^{-1}(\cdot)$ the inverse of $D(\cdot)$, any assigned constraint: $D(\theta) \leq D_{\max }$ on the subjective QoS level to be guaranteed may be equivalently recast in terms of the corresponding constraint: $\theta(t) \geq \theta_{\min } \triangleq D^{-1}\left(D_{\max }\right)$ on the minimum rate to be instantaneously delivered by the playout buffer.

This constraint guarantees that the maximum (e.g., worst-case) queueing delay induced by the playout buffer of Fig 1 equates: $\left(N_{2} / \theta_{\min }\right)$, so that, for the corresponding playout-delay r.v. $\boldsymbol{T}_{Q Q}$ (e.g., the random delay introduced by the playout buffer), the following deterministic (e.g., hard) upper-bound holds:

$$
\boldsymbol{T}_{Q Q} \leq\left(N_{2} / \theta_{\min }\right) \quad(\text { slot }) .
$$

Passing now to consider the performance limit on the jitter affecting the playout rate, we observe that both the instantaneous jitter and corresponding average squared value become upper-bounded when the following limit on the maximum allowed playout rate is also introduced: $\theta(t) \leq \theta_{\max }, \forall t$. In fact, the above constraints guarantee that the resulting playout-jitter is limited (in a hard way) as detailed by the following Proposition 1], proved in [10].

Proposition 1. The instantaneous jitter affecting the playout rate is upperbounded as in $\left|\theta\left(t_{1}\right)-\theta\left(t_{2}\right)\right| \leq\left(\theta_{\max }-\theta_{\min }\right), \forall t_{1} \gtreqless t_{2}$. Furthermore, in the steady-state, the corresponding average playout jitter: $\sigma_{\theta} \triangleq \sqrt{E\left\{(\boldsymbol{\theta}(t)-\bar{\theta})^{2}\right\}}$ $(I U /$ slot $)$ is limited as in $\sigma_{\theta} \leq \sqrt{\left(\theta_{\max }\right)^{2}-\left(\theta_{\min }\right)^{2}}$.

To recap, the considered constraints suffice to guarantee that the performance limits of the streaming system of Fig 1 are dictated by eqs.(4) and upper-bounds provided by Proposition 1 .

\section{The Tackled STE Problem}

In our framework, the overall system's state $\underline{x}(t)$ available at slot t-th for implementing the resulting scheduling action:

$$
\underline{x}(t) \triangleq[\sigma(t), s(t), q(t), r(t-1), r(t-2), \ldots, r(t-p))]^{T},
$$


is composed by the current connection's state $\sigma(t)$ (see Fig 1), the current back$\log s(t)$ of the playin buffer, the current backlog $q(t)$ of the playout buffer and the last $p \geq 0$ transmit rates: $r(t-1), \ldots, r(t-p)$.

Hence, to formally state the resulting STE problem, let: $\mathcal{E}(t) \equiv \varepsilon(\sigma(t) ; r(t)) \triangleq$ $\mathcal{R}^{-1}(\sigma(t) ; r(t)), t \geq 1$, be the energy to be radiated by the wireless modem present at the PHY layer of the SH of Fig 1 to send $r(t)$ IUs. Therefore, the tackled STE problem may be stated as follows:

$$
\begin{gathered}
\max _{[r(\cdot), \lambda(\cdot), \theta(\cdot)]} \mathrm{E}_{\sigma}\{\mathbf{r}(t) \mid s(t), q(t)\}, \\
\text { s.t. : } \mathrm{E}_{\sigma}\{\varepsilon(\boldsymbol{\sigma} ; r(t)) \mid s(t), q(t)\} \leq \mathcal{E}_{\text {ave }}, \\
0 \leq r(t) \leq s(t), \\
0 \leq \lambda(t) \leq \lambda_{\max }, \\
0 \leq s(t+1) \leq N_{1}, \\
N_{0} \leq q(t+1) \leq N_{2}, \\
\theta_{\min } \leq \theta(t) \leq \theta_{\max },
\end{gathered}
$$

where the expectations in (6) and (7) are to be carried out with respect to the (a priori unknown) pdf $p_{\sigma}(\sigma)$ of the connection's state. Hence, from the outset it follows that the playout function $\theta(t)$ to be optimized may be directly expressed as in (see (5) ): $\theta(t) \equiv \mathcal{F}(r(t), r(t-1), \ldots, r(t-p)), t \geqslant 1$, where $\mathcal{F}(\cdot)$ is a suitable nonnegative real-valued function that, in general, may depend on the current transmit rate $r(t)$ and $p$ last ones: $r(t-1), \ldots, r(t-p)$. This points out that $p$ plays the role of memory-order of the implemented playout policy.

Before proceeding, we remark that the stochastic nature of the considered TE problem arises from the expectations present in eqs.(6),(17). In this regard, eq.(6) points out that we maximize (on a per slot-basis) the expected (e.g., average) transmit rate given (i.e., conditioned on) the current backlogs: $(s(t), q(t))$ of the playin and playout buffers. Several contributions (see, for example, [14, Chaps.13,17] and references therein) support the utilization of (6) as an effective metric for characterizing the performance of queuing systems, specially when the supported media traffics exhibit heavy-tailed (e.g., Pareto-like) distributions. Finally, from (11),(12), it follows that the ratio: $\Delta_{P} \triangleq N_{0} / \theta_{\min }$ covers the role of allowed pre-roll delay.

\subsection{Algorithmic Solution of the Tackled STE Problem}

Let us indicate by $\varepsilon_{r}(r ; \sigma) \triangleq \partial \varepsilon(r ; \sigma) / \partial r$ the first-order derivative of the (above introduced) energy-function carried out with respect to the $r$-argument. Thus, after recognizing that the tackled STE problem is convex [10, the resulting optimal solution: $r^{o p t}(\cdot ; \cdot ; \cdot), \lambda^{o p t}(\cdot ; \cdot ; \cdot), \theta^{o p t}(\cdot ; \cdot ; \cdot)$ may be evaluated in closedform, as detailed by the following Proposition 2 (proved in [10]).

Proposition 2. Under the above reported assumptions, for the solution of the STE problem of eqs.(6)-(12) the following relationships hold: 
i) the optimal transmit rate is dictated by

$$
r^{o p t}(\sigma(t) ; s(t) ; q(t))=\left[\varepsilon_{r}^{-1}\left(\sigma ; \frac{1}{\mu\left(s(t) ; q(t) ; M_{p}(t)\right)}\right)\right]_{r_{m}\left(M_{p}(t) ; s(t) ; q(t)\right)}^{r_{p}\left(M_{p}(t) ; s(t) ; q(t)\right)}
$$

where $\varepsilon_{r}^{-1}(\cdot ; \cdot)$ denotes the inverse function of $\varepsilon_{r}(\cdot ; \cdot)$ with respect to the $\mathcal{E}$ variable, while

$r_{m}\left(M_{p}(t) ; s(t) ; q(t)\right) \triangleq \max \left\{0 ;(p+1) \theta_{\min }-p M_{p}(t) ;\left(\frac{p+1}{p}\right)\left(N_{0}-q(t)\right)+M_{p}(t)\right\}$

and

$r_{p}\left(M_{p}(t) ; s(t) ; q(t)\right) \triangleq \min \left\{s(t) ;\left(\frac{p+1}{p}\right)\left(N_{2}-q(t)\right)+M_{p}(t) ;(p+1) \theta_{\max }-p M_{p}(t)\right\}$

are the minimum and peak-rate values allowed at slot $t$, respectively. Furthermore,

$$
M_{p}(t) \triangleq \frac{1}{p} \sum_{i=t-p}^{t-1} r^{o p t}(\sigma(i) ; s(i) ; q(i))
$$

represents the short-term average of the (optimal) transmit rates. Finally, $\mu(\cdot ; \cdot ; \cdot)$ in (13) is the optimal value of the dual variable of the tackled optimization problem, that may be computed by solving the following equation [10]:

$$
\int \varepsilon\left(\sigma ;\left[\varepsilon_{r}^{-1}\left(\sigma ; \frac{1}{\mu\left(s(t) ; q(t) ; M_{p}(t)\right)}\right)\right]_{r_{m}(\cdot ; ; \cdot)}^{r_{p}(\cdot ; \cdot ; \cdot)}\right) p_{\sigma}(\sigma) d \sigma=\mathcal{E}_{\text {ave }} .
$$

ii) The optimal playin-rate policy is given by the following relationship:

$$
\lambda^{o p t}(\sigma(t) ; s(t) ; q(t))=\min \left\{\left(N_{1}-s(t)\right)+r^{o p t}(\sigma(t) ; s(t) ; q(t)) ; \quad \lambda_{\max }\right\} .
$$

iii) The optimal playout-rate policy is dictated by following relationship:

$$
\theta^{o p t}(\sigma(t) ; s(t) ; q(t))=r^{o p t}\left(M_{p}(t) ; s(t) ; q(t)\right)+\frac{p}{p+1}\left(M_{p}(t)-r^{o p t}\left(M_{p}(t) ; s(t) ; q(t)\right)\right)
$$

Before proceeding, we stress that a key property of the optimal scheduler of Proposition 2 is that it utilizes the steady-state pdf $p_{\sigma}(\sigma)$ of the wireless connection only for the computation of the expectation at the left-hand side of (15). Therefore, an approximated version $\widehat{\mu}($.$) of \mu($.$) in (15) may be computed by solv-$ ing (on a per slot-basis) the following sliding-window sample-average equation:

$$
\frac{1}{W} \sum_{j=t-W+1}^{t} \varepsilon\left(\sigma(j) ;\left[\varepsilon_{r}^{-1}\left(\sigma(j) ; \frac{1}{\widehat{\mu}(t)}\right)\right]_{r_{m}(\cdot ; \cdot ; \cdot)}^{r_{p}(\cdot ; \cdot ; \cdot)}\right)=\mathcal{E}_{\text {ave }}, \quad t \geq 1,
$$


where, due to the (assumed) system's ergodicity, we have that: $\lim _{W \longrightarrow \infty} \widehat{\mu}(t)=$ $\mu(t)$. Thus, by resorting to (18), the optimal scheduler may be implemented on-the-fly, i.e., without any a priori knowledge about actual $p_{\sigma}(\sigma)$. This implies that the on-the-fly implementation of the optimal scheduler is also capable to self-track a priori unknown possible time-variations of the pdf $p_{\sigma}(\sigma)$ of the connection-state, as those induced, for example, by no stationary fading.

\section{Traffic Analysis of IP-over-Wireless Access Connections}

Since the access segment is typically the "bottleneck" of current wireless media networks [12], in order to test actual performance of the proposed joint scheduler, in this section we develop the traffic analysis of a wireless access network, where a (possibly nomadic) wireless SH transmits to a (possibly wireless and/or nomadic) DH via a Wireless Access Router (WAR). This last may be simultaneously utilized for the access by other several concurrent Interfering Hosts (IHs), that, in turns, may cause interference and congestion (e.g., buffers' overflow) at the PHY and Network layers of the WAR. The resulting layered structure of the considered protocol stack is detailed in Fig,2. Specifically, FEC-modulation supported by adaptive control of the transmit energy is implemented at the PHY layers of the SH and WAR of Fig[2. The Data Link (DL) layer of the SH node of Fig 2 is equipped with a finite-capacity buffer, so to implement a Truncated Selective Repeat ARQ (TSR-ARQ) mechanism [12, Chap.5]. At most, $N_{R}^{\max } \geq 0$ re-transmissions of a same frame may be attempted at the DL layer, before declaring lost the served frame.

Before proceeding to the goodput analysis of the resulting wireless pipe of Fig[2, we detail the considered main operating conditions:

$\boldsymbol{A} \boldsymbol{i}$ ) the forward wireless channel of Fig 2 is frequency-flat and impaired by both i.i.d. Nakagami-distributed fast-fading and log-normal distributed slowfading [12. Specifically, the (log-normal distributed) slow-fading phenomena are assumed constant over at least $\mathcal{X} \triangleq\left(N_{R}^{\max }+1\right)$ consecutive slot-times, and then may vary according to the Markovian model detailed in the sequel; $\boldsymbol{A}$.ii) when a frame is received incorrectly at the DL layer of DH after FEC/re-transmission recovery, both the corresponding encapsulated datagram and segment are declared loss. Then, triple-duplicate ACKs are generated by the Transport layer of the DH and sent back to the Transport layer of the SH via the ideal backward link of Fig 2, Furthermore, due to the real-time feature of the considered streaming applications, no fragmentation is allowed at the Transport and DL layers of the protocol stack of Fig 2, neither timeout-triggered re-transmissions are assumed feasible at the Transport layer of the SH of Fig $1 \boldsymbol{A . i i i )}$ due to implementation complexity considerations [12], the energy $\mathcal{E}(t)$ radiated by the transmitter at the PHY layer of the $\mathrm{SH}$ of Fig 2 is assumed to react only to the fluctuations induced by slow-fading. Thus, according to $\boldsymbol{A}$.i), the transmit energy $\mathcal{E}(t)$ is assumed constant over (at least) $\mathcal{X}$ consecutive slot-times.

For flat-fading wireless channels meeting $\boldsymbol{A} . \boldsymbol{i}$ ), the channel quality experienced over each slot may be described by the corresponding instantaneous 


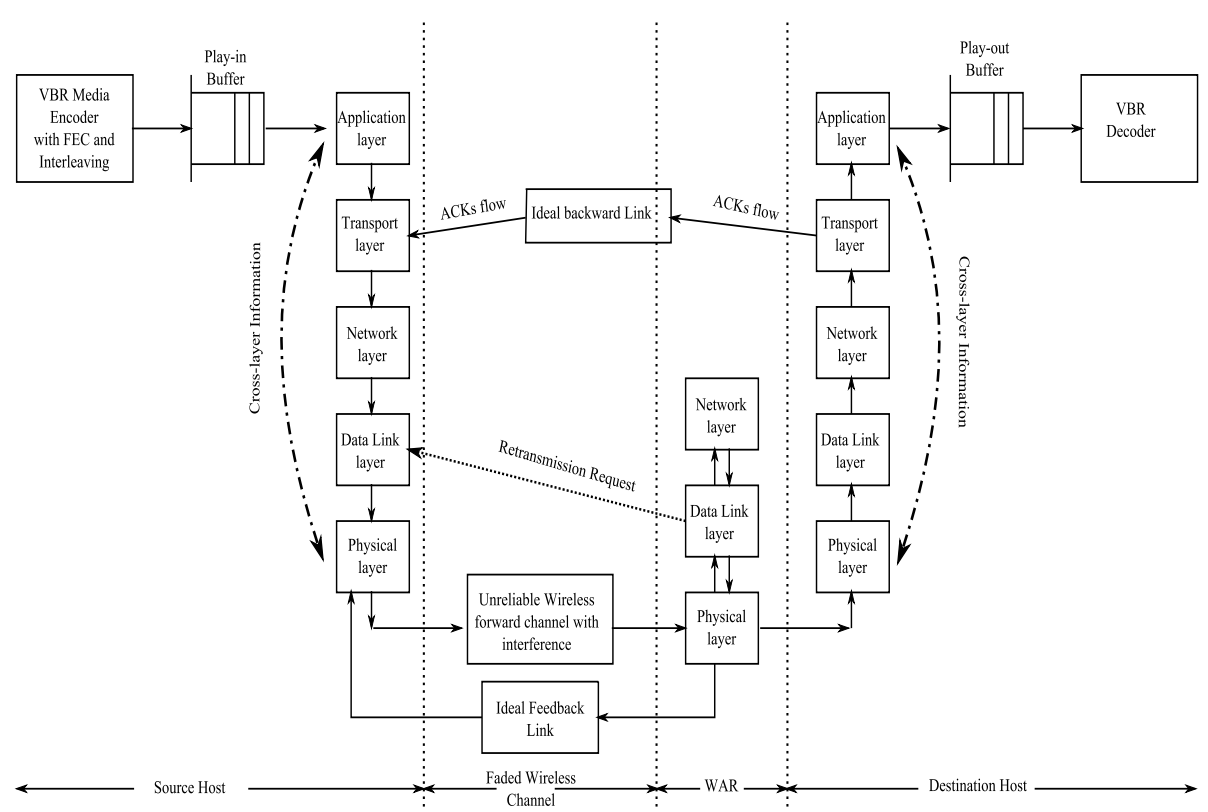

Fig. 2. The layered structure of the considered access network

channel gain $g(t) \in \mathbb{R}_{0}^{+}$. In our case, this last is Nakagami-m distributed, so that, for the resulting pdf $p(g)$, we can write [12, Chap.3]

$$
p(g)=(m / \bar{g}(t))^{m}\left(g^{m-1} / \Gamma(m)\right) \exp \{-(m / \bar{g}(t)) g\}, \quad g \geq 0,
$$

where $\Gamma(m)$ is the Gamma function and $m \geq 1 / 2$ is the Nakagami fading parameter. By definition, $\bar{g}(t)$ in (19) is the instantaneous SINR averaged over (only) the fast-fading phenomena. Hence, for log-normal distributed slow-fading, it may be modeled as [12, Chap.3] where the scaling constant: $c_{0} \triangleq \exp \left\{-\frac{(0.1 \log 10)^{2}}{2}\right\} \approx$ 0.9738 guarantees unit average value for $\overline{\boldsymbol{g}}(t)$, e.g, $\bar{\gamma} \triangleq E\{\overline{\mathbf{g}}(t)\} \equiv 1\left(\mathrm{~J}^{-1}\right)$. Furthermore, according to $12,\{z(t), t \geq 1\}$ is a zero-mean unit-variance Gaussiandistributed Markovian sequence, that assumes constant value over each frame composed by $\mathcal{X}$ consecutive time-slots (see $\boldsymbol{A}$. $\boldsymbol{i}$ )). For macrocellur land-mobile applications, its correlation coefficient: $h \triangleq E\{z(t) z(t+\mathcal{X})\}$ may be evaluated as in [12]: $h \equiv(0.82)^{T_{S} v / 100}$, where $v(\mathrm{~m} / \mathrm{sec}$. $)$ is the speed of the SH node of Fig,2.

Thus, according to the performance analysis carried out in [9], the instantaneous Frame Error Rate (FER) measured at the input of the DL layer of the WAR of Fig 2 after FEC-recovery may be well approximated according to 9 , eq. (5)]

$$
F E R(t) \approx \begin{cases}1, & \text { for } 0 \leq g(t)<C / \mathcal{E}(t) \\ A \exp \{-B \mathcal{E}(t) g(t)\}, & \text { for } g(t) \geq C / \mathcal{E}(t)\end{cases}
$$


where the (positive) constants $A, B, C$ in (20) depend on the actually adopted coding/modulation scheme [9].

Therefore, after frame recovery carried out by the Truncated SR-ARQ mechanism implemented at the DL layer, the resulting peer-to-peer IP-based connection available at the Network layer of Fig 2 may be modeled as a lossaffected pipe, that may also introduces random delays on the conveyed datagrams' flow 7. Specifically, according to the delay-analysis presented in 7, Sect.II], we model the sequence $\{\Delta(\mathcal{X} t), t \geq 1\}$ of datagram's delays as an i.i.d. exponentially-distributed random series, so that the resulting $\operatorname{pdf} p_{\Delta}(\Delta)$ for the datagram delay reads as (see [7, Sect.II] for the case of a single access router): $p_{\Delta}(\Delta)=\varepsilon_{L} \delta(\Delta-\infty)+\left(1-\varepsilon_{L}\right) \omega_{R} e^{-\omega_{R} \Delta}, \Delta \geq 0$, where $\delta(\Delta-\infty)$ is the Dirac pulse shifted at the infinity, $\omega_{R}>0$ is the service rate (in $(\text { slot })^{-1}$ ) of the WAR of Fig 2 , while $\varepsilon_{L} \in[0,1)$ is the overall datagram loss-rate affecting the wireless pipe of Fig, 2 ,

Finally, under low loss-rate operating conditions, the sending rate $\mathcal{R}(\mathcal{X} t)$ (byte/slot) of the TCP-friendly rate-control mechanism implemented at the Transport layer of the $\mathrm{SH}$ node of Fig 2 may be well approximated by the following expression [11]:

$$
\left.\left.\mathcal{R}(\mathcal{X} t) \equiv\left[M S S / \overline{R T T}(\mathcal{X} t) \sqrt{\overline{P_{L}}(\mathcal{X} t)}\right)\right] \sqrt{3 / 4}, t \geq 0, \text { (byte/slot }\right),
$$

where $M S S$ (byte) is the allowed maximum segment size; $\overline{P_{L}}(\mathcal{X} t)$ is the segmentloss probability averaged over the fast-fading statistics of eq.(19); and $\overline{R T T}(\mathcal{X} t)$ is the average segment Round-Trip-Time (RTT) at the $(\mathcal{X} t)$-th slot. According to the Jacobson's formula, this last may be iteratively updated as in $\overline{R T T}(\mathcal{X} t) \equiv$ $0.75 \overline{R T T}(\mathcal{X}(t-1))+0.25 \Delta(\mathcal{X} t), t \geq 1$.

About the evaluation of the resulting connection goodput, we stress that, in our framework, the reception of triple-duplicate ACKs is only utilized for updating the current value of $\bar{P}_{L}(\cdot)$ in (21). This means that the here considered Transport protocol is, indeed, UDP based, but it relies on a suitable traffic shaper to work in a TCP-friendly way [11. Thus, by leveraging on this (key) observation, we are able to arrive at the following final expression for the goodput in (21) sustained by the considered connection (see [10] for the derivation):

$$
\mathcal{R}(\mathcal{X} t)=K_{0} \sigma(\mathcal{X} t)(\mathcal{E}(\mathcal{X} t))^{\mathcal{X} m / 2}, \quad t \geq 0
$$

where $K_{0} \triangleq \sqrt{3 / 4} M S S\left\{\left(m^{m} / \Gamma(m)\right)\left[\left(C^{m} / m\right)+(A \Gamma(m ; C B)) / B^{m}\right]\right\}^{-\mathcal{X} / 2}$, is a positive constant, while,

$$
\sigma(\mathcal{X} t) \triangleq[\bar{g}(\mathcal{X} t)]^{\mathcal{X} m / 2} / \overline{R T T}(\mathcal{X} t), \quad t \geq 0,
$$

is the (positive-valued) resulting state of the overall peer-to-peer connection going from the input of the Transport layer at the SH of Fig 2 to the output of the Transport layer at the corresponding DH. Hence, under the above reported assumptions, eq.(22) is able to fully characterize the goodput-behavior of the overall resulting TCP-friendly connection. 


\section{$5 \quad$ Traffic Tests and Final Remarks}

According to this conclusion, in order to (numerically) test actual performance of the proposed joint scheduler, we have numerically generated the connectionstate sequence $\{\sigma(\mathcal{X} t)\}$ of eq. (23) and, then, we have adopted the expression in (22) for (numerically) measuring the instantaneous goodput $\mathcal{R}(\cdot ; \cdot)$ sustained by the overall peer-to-peer pipe. The main simulated parameters are set as $\lambda_{\max }=$ $\theta_{\max }=1000$ (byte/slot), $M S S=120$ (byte), $N_{0}=0$ (byte), $A=90.2514$, $B=3.4998, C=1.2865, \mathcal{X} \equiv 1, b=2$ and $N_{1}=N_{2}=24000$ (byte).

\subsection{Effects of the Memory-Order of the Playout Policy}

Performance of the self-adaptive implementation of the proposed scheduler has been numerically evaluated in terms of average playout rate: $\bar{\theta}^{\text {opt }} \triangleq E\left\{\boldsymbol{\theta}^{\text {opt }}(t)\right\}$ (byte/slot) and average playout rate-jitter: $\sigma_{\theta}^{o p t} \triangleq \sqrt{E\left\{\left(\boldsymbol{\theta}^{\text {opt }}-\bar{\theta}^{\text {opt }}\right)^{2}\right\}}$ (byte/slot). For comparison purpose, we have also implemented a Benchmark Version (BV) of the scheduler of Sect 3, where both the transmit-energy and playout-rate controls have been removed. The (numerically evaluated) behaviors of the average playout rate $\bar{\theta}_{B V}$ (byte/slot) and playout rate-jitter $\sigma_{\theta}^{B V}$ (byte/slot) we obtained by implementing this BV are reported in Figs $3(\mathrm{a})[3(\mathrm{~b})$, respectively. For comparison purpose, Figs $3(\mathrm{a})[3(\mathrm{~b})$ also report the corresponding performance of the self-adaptive implementation of the optimal scheduler. All the numerical plots of Figs $3(\mathrm{a})[3(\mathrm{~b})$ refer to the case of $\omega_{R}=1\left(\right.$ slot $\left.^{-1}\right), \varepsilon_{L}=0, m=1$, and $W=5$ (slot).

An examination of these plots allows us to drawn the following insights. First, $\bar{\theta}^{\text {opt }}$ is virtually independent from the setting of the memory order $p$ adopted for the playout policy. Second, the gap between $\bar{\theta}^{\text {opt }}$ and $\bar{\theta}_{B V}$ tends to decrease

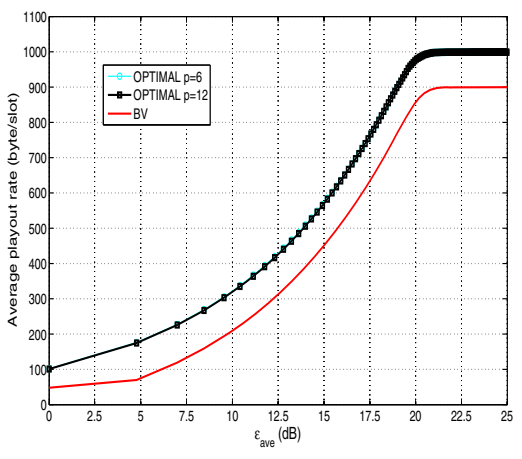

(a)

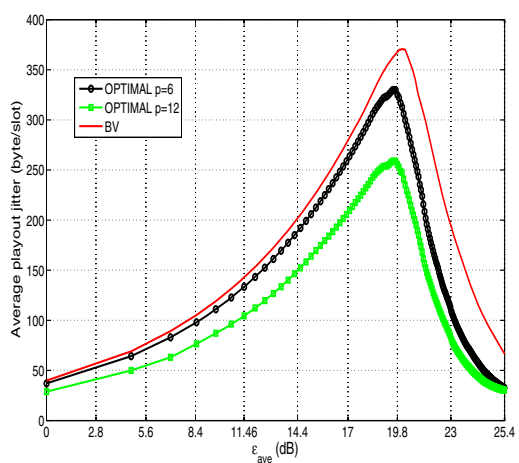

(b)

Fig. 3. Behaviors of: (a) the average playout rates $\bar{\theta}^{\text {opt }}$ (byte/slot) and $\bar{\theta}_{B V}$ (byte/slot); (b) the average playout jitters $\sigma_{\theta}^{o p t}$ (byte/slot) and $\sigma_{\theta}^{B V}$ (byte/slot). Plots of $\bar{\theta}^{\text {opt }}$ at $p=6$ and $p=12$ are virtually overlapped. 


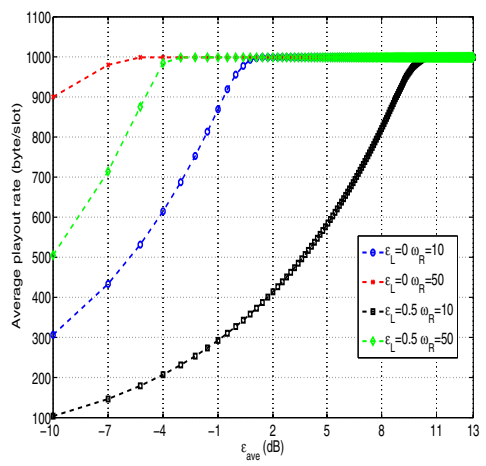

(a)

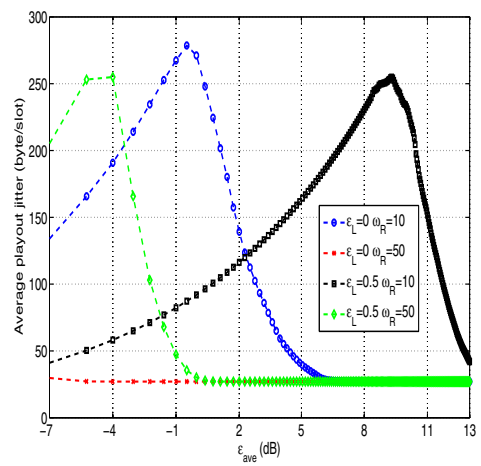

(b)

Fig. 4. Behaviors of: (a) the average playout rate $\bar{\theta}^{\text {opt }}$ (byte/slot); and: (b) the average playout jitter $\sigma_{\theta}^{o p t}$ (byte/slot) of the optimal scheduler under several traffic conditions. The operating scenario of Sect 5.2 is considered.

at both low and high $\mathcal{E}_{\text {ave }}$ 's, while it is noticeable and of the order of about $45 \%$ at $\mathcal{E}_{\text {ave }}$ 's around $10(d B)$. Third, jitter curves of Fig $3(\mathrm{~b})$ point out that, at low/medium $\mathcal{E}_{\text {ave }}$ 's (we say, at $\mathcal{E}_{\text {ave }}$ 's limited up to about $19(d B)$ ), the average jitters affecting the played rates tend to increase for growing $\mathcal{E}_{\text {ave }}$ 's. However,

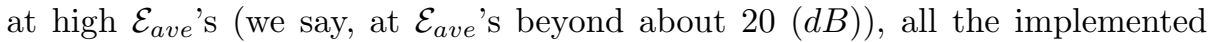
transmit policies become able to fully saturate the capacities of the corresponding playout buffers, so that all the resulting jitters tend to decrease (see the rightmost parts of the curves of Fig $3(\mathrm{~b})$. In any case, the practical lesson arising from

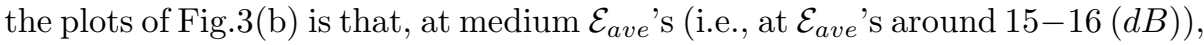
the optimal scheduler with $p=12$ is able to reduce the average jitter affecting the corresponding playout rate of about $33 \%$ with respect to the $\mathrm{BV}$.

\subsection{Effects of Traffic Load and Traffic Congestion}

The average performances of the on-the-fly implementation of the optimal scheduler under different traffic load and traffic congestion levels are reported in Figs $4(\mathrm{a}) 4(\mathrm{~b})$ for the case of $m=1, W=5$ (slot), $p=12$ and $\theta_{\text {min }}=$ 100 (byte/slot). An examination of these curves shows that the relative degradations suffered by $\bar{\theta}^{o p t}$ and $\sigma_{\theta}^{o p t}$ when we pass from: $\varepsilon_{L}=0, \omega_{R}=50$ (i.e., the less congested traffic scenario here considered) to: $\varepsilon_{L}=0.5, \omega_{R}=10$ (i.e., the most congested traffic scenario here considered) are noticeable only at (very) low

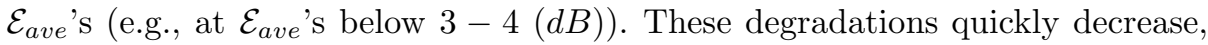
indeed, for $\mathcal{E}_{\text {ave }}$ 's beyond $4-5(d B)$ and, then, virtually vanish at $\mathcal{E}_{\text {ave's over }}$ about $9(d B)$.

\section{References}

1. Van der Schaar, M., Chou, P.A.: Multimedia over IP and Wireless Networks. Academic Press, London (2007) 
2. Mitra, D., Wang, Q.: Stochastic Traffic Engineering for Demand Uncertainty and Risk-Aware Network Revenue Management. IEEE/ACM Tr. Networking 13(2), 221-233 (2005)

3. Wang, H., Xie, H., Qui, L., Yang, Y.R., Zhang, Y., Greenberg, A.: Cope: Traffic Engineering in Dynamic Networks. In: Proc. ACM Sigcomm (2006)

4. Yan, L., Markopoulou, A., Bambos, N., Apostolopoulos, J.: Joint power-playout control for media streaming over wireless links. IEEE Tr. on Multimedia 8(4), 830-843 (2006)

5. Sawma, G., Ben-El-Kezadri, R., Aib, I., Bezalel, G., Pujolle, G.: Proactive Traffic Engineering for IEEE 802.11 Mobile Wireless Networks. In: IEEE VTC 2009-Fall (September 2009)

6. Faruque, S.: Traffic Engineering for multi-rate wireless data. In: IEEE International Conference on Electro/Information Technology, pp. 280-283 (May 2008)

7. Chou, P., Miao, Z.: Rate-distortion optimized streaming of packetized media. IEEE Tr. on Multimedia 8(2), 390-404 (2006)

8. Kalman, M., Steinbach, E., Girod, B.: Adaptive media playout for low-delay streaming over error-prone channels. IEEE Tr.on Circuits and Systems for Video Technology 14(6), 841-851 (2004)

9. Liu, Q., Zhou, S., Giannakis, G.B.: Cross-layer combining of adaptive modulation and coding with truncated ARQ over wireless links. IEEE Tr. on Wireless Communications 3(5), 1746-1755 (2004)

10. Cordeschi, N., Patriarca, T., Baccarelli, E.: Energy-Efficient adaptive playin/playout providing hard QoS guarantees to wireless media-streaming applications, DIET Techn. Report, available at the web site, http://infocom.uniroma1.it/ enzobac/playout_technicalreport.pdf

11. Jin, S., Matta, I., Bestravos, A.: A Spectrum of TCP-Friendly Window-Based Congestion Control algorithms. IEEE/ACM Tr. on Networking 11(3), 341-355 (2003)

12. Glisic, S.G.: Advanced Wireless Networks-4G Technologies. Wiley, Chichester (2006)

13. Chuang, H.C., Huang, C.Y., Chiang, T.: Content-aware Adaptive Media Playout Controls for wireless video streaming. IEEE Tr. on Multimedia 9(6), 1273-1283 (2007)

14. Park, K., Willinger, W.: Self-Similar Networks Traffic and Performance Evaluation. Wiley, Chichester (2000) 